\title{
A Non-linear Lower Bound for Planar Epsilon-nets
}

\author{
Noga Alon
}

Received: 19 September 2010 / Revised: 2 December 2010 / Accepted: 6 December 2010 /

Published online: 5 January 2011

(C) Springer Science+Business Media, LLC 2011

\begin{abstract}
We show that the minimum possible size of an $\epsilon$-net for point objects and line (or rectangle)-ranges in the plane is (slightly) bigger than linear in $\frac{1}{\epsilon}$. This settles a problem raised by Matoušek, Seidel and Welzl (Proc. 6th Annu. ACM Sympos. Comput. Geom., pp. 16-22, 1990).
\end{abstract}

Keywords Epsilon nets · Weak epsilon nets · VC-dimension

\section{Introduction}

A range space $S$ is a pair $(X, R)$, where $X$ is a (finite or infinite) set and $R$ is a (finite or infinite) family of subsets of $X$. The members of $X$ are called points and those of $R$ are called ranges. If $A$ is a subset of $X$ then $P_{R}(A)=\{r \cap A: r \in R\}$ is the projection of $R$ on $A$. In case this projection contains all subsets of $A$, we say that $A$ is shattered. The Vapnik-Chervonenkis dimension (or VC-dimension) of $S$, denoted by $V C(S)$, is the maximal cardinality of a shattered subset of $X$. If there are arbitrarily large shattered subsets then $V C(S)=\infty$.

For a finite set of points $A$ in a range space, a subset $N \subset A$ is an $\epsilon$-net for $A$ if any range $r \in R$ satisfying $|r \cap A| \geq \epsilon|A|$ contains at least one point of $N$. The theory

A preliminary version of this paper appeared in the 51st Annual IEEE Symposium on Foundations of Computer Science (FOCS 2010).

Research supported in part by an ERC Advanced grant, by the Hermann Minkowski Minerva Center for Geometry in Tel Aviv University, by the Oswald Veblen Fund and by the Bell Companies

Fellowship.

N. Alon ( $\varangle)$

Schools of Mathematics and Computer Science, Sackler Faculty of Exact Sciences, Tel Aviv

University, Tel Aviv 69978, Israel

e-mail: nogaa@tau.ac.il

N. Alon

Institute for Advanced Study, Princeton, NJ 08540, USA 
of VC-dimension and $\epsilon$-nets has played a central role in discrete and computational geometry, and has been used in a variety of applications including range searching, geometric partitions, and bounds on various incidence problems, as well as in other mathematical areas such as statistics, computational learning, discrepancy theory and combinatorics.

A well known result of Haussler and Welzl [20], following earlier work of Vapnik and Chervonenkis [34], asserts that for any $n$ and $\epsilon>0$, any set of size $n$ in a range space of VC-dimension $d$ contains an $\epsilon$-net of size at most $O\left(\frac{d}{\epsilon} \log \left(\frac{1}{\epsilon}\right)\right)$. See also [22] for a proof of a $(1+o(1))\left(\frac{d}{\epsilon} \log \left(\frac{1}{\epsilon}\right)\right)$ upper bound, and [3, 24, 27] for more details.

As shown in [22], there are known constructions in which for fixed $d$ the size of the smallest possible $\epsilon$-net for a given set cannot be linear in $\frac{1}{\epsilon}$. In fact, the $O\left(\frac{d}{\epsilon} \log \left(\frac{1}{\epsilon}\right)\right)$ bound may be tight already for dimension $d=2$, as shown in [22] (see also [6] for another construction). Despite the existence of these constructions, there is no known natural geometric example demonstrating this phenomenon. Indeed, there is no known lower bound, better than the trivial $\Omega\left(\frac{1}{\epsilon}\right)$ bound, in any concrete geometric situation. The problem, raised and addressed 20 years ago in [26], whether or not in all natural geometric scenarios of VC-dimension $d$, there always exists an $\epsilon$-net of size $O\left(\frac{d}{\epsilon}\right)$, is still wide open, and has been considered in a substantial number of papers over the years. Besides being interesting in its own right, this question has an algorithmic motivation, since as shown in [8] and [14] the existence of smaller nets supplies improved approximation algorithms for the set cover problem and the hitting set problem in the corresponding geometric scenarios.

A linear (in $\frac{1}{\epsilon}$ ) upper bound for the size of $\epsilon$-nets has been established for several special geometric cases, such as point objects and halfspace ranges in two and three dimensions, and point objects and disk or pseudo-disk ranges in the plane; see [1, 2, $10,12,23,26,28]$ and the survey [15] for some earlier results on the subject. See also [13, 19, 32] and [7] for more recent results, and [21, 35] for additional improved (though not quite linear) bounds for $\epsilon$-nets in geometric settings. As mentioned in many of these papers, the prevailing conjecture so far has been that in all geometric scenarios, there always exists an $\epsilon$-net of size $O\left(\frac{d}{\epsilon}\right)$.

\subsection{The New Results}

In the present short paper, we show that the linear bound does not hold, and the smallest possible size of an $\epsilon$-net in a very simple geometric situation (with VCdimension 2) is not linear. Unfortunately, our lower bound is only barely non-linear, providing planar geometric examples in which the minimum size of an $\epsilon$-net is at least $\Omega\left(\frac{1}{\epsilon} w\left(\frac{1}{\epsilon}\right)\right)$, where $w$ is (a version of) the inverse Ackermann function. It is worth noting that if the VC-dimension is $d=1$ there are always $\epsilon$-nets of size $O\left(\frac{1}{\epsilon}\right)$. This is proved (in a precise form) in [28] and also follows from the result in [4] that any range space of $\mathrm{VC}$-dimension 1 can be embedded in a range space of half-spaces in dimension at most 3 . Therefore, the dimension 2 in our example is optimal.

For convenience, we state in the following two theorems the main results demonstrating the non-linear behavior of the functions, without specifying the precise bounds obtained. 
Theorem 1.1 For every (large) positive constant $C$ there exist $n$ and $\epsilon>0$ and a set $X$ of $n$ points in the plane, so that the smallest possible size of an $\epsilon$-net for lines for $X$ is larger than $C \cdot \frac{1}{\epsilon}$.

The construction in the proof of this theorem provides examples in which $\epsilon$ is not much bigger than $1 / n$. It seems more interesting to find examples in which for each fixed $\epsilon$ in a decreasing sequence, $n$ can be arbitrarily large. This is done in the next theorem.

A fat line in the plane is the set of all points within distance $\mu$ from a line in the plane. Equivalently, this is the intersection of two half planes with parallel supporting lines. Our construction implies the following.

Theorem 1.2 For every (large) positive constant $C$ there exists a sequence $\epsilon_{i}$ of positive reals tending to zero, so that for every $\epsilon=\epsilon_{i}$ in the sequence and for all $n>n_{0}\left(\epsilon_{i}\right)$ there exists a set $Y_{n}$ of $n$ points in general position in the plane, so that the smallest possible size of an $\epsilon$-net for fat lines for $Y_{n}$ is larger than $C \cdot \frac{1}{\epsilon}$.

It is not difficult to check that the VC-dimension of the range space considered in the first theorem is 2 , while that of the space considered in the second is 5 .

\subsection{Weak $\epsilon$-nets}

Our bounds hold for weak $\epsilon$-nets as well. For a finite set of points $X$ in $R^{m}$ and a (possibly infinite) family of subsets $\mathcal{F}$ of $R^{m}$, a set $Y \subset R^{m}$ is a weak $\epsilon$-net for $X$ with respect to $\mathcal{F}$ if any $F \in \mathcal{F}$ that satisfies $|F \cap X| \geq \epsilon|X|$ contains at least one point of $Y$. The difference between this notion and that of a (strong) $\epsilon$-net considered in the previous subsection is that here $Y$ does not have to necessarily be a subset of $X$. Indeed, this makes the task of finding a small net much easier, and unlike the case of strong nets it is known that there is a function $f(\epsilon, m)$ depending only on $\epsilon$ and $m$ so that for every finite set $X$ (of any size) in $R^{m}$, there is a weak $\epsilon$-net $Y$ for $X$ with respect to the set of all convex sets in $R^{m}$, where $|Y| \leq f(\epsilon, m)$. This was first proved in [5], see also [11] and [25] for improved bounds, and [6] and its references for several extensions. The corresponding assertion for strong nets is easily seen to be false (and indeed the VC-dimension of the family of all convex sets is infinite, in every fixed dimension $m \geq 2$ ). The best known upper bound for the function $f(\epsilon, 2)$ is $O\left(\frac{1}{\epsilon^{2}}\right)$, proved in [5], and until recently there was no known lower bound exceeding $\Omega\left(\frac{1}{\epsilon}\right)$. Such a bound (of $\Omega\left(\frac{1}{\epsilon} \log \left(\frac{1}{\epsilon}\right)\right.$ ) was proved in [9] using an elegant technique based on a notion the authors call stair convexity.

The results in Theorem 1.1 and in Theorem 1.2 can be extended to weak nets as well. Although this provides much weaker lower bounds than the above mentioned $\Omega\left(\frac{1}{\epsilon} \log \left(\frac{1}{\epsilon}\right)\right)$ bound of [9], these bounds are still non-linear in $\frac{1}{\epsilon}$ and hold for a very restricted collection of convex sets: lines, fat lines, rectangles or triangles, all having bounded VC-dimension. In this respect, this is stronger than the results in [9]. For convenience, as done for the case of strong nets, we state the non-linear lower bound for the size of weak nets too without specifying the precise estimate the proof provides. 
Theorem 1.3 For every (large) positive constant $C$ there exist $n$ and $\epsilon>0$ and a set $X$ of $n$ points in the plane, so that the smallest possible size of a weak $\epsilon$-net for lines for $X$ is larger than $C \cdot \frac{1}{\epsilon}$.

The analog of Theorem 1.2 for weak nets holds as well.

\subsection{Organization}

The rest of this short paper is organized as follows. In Sect. 2, we describe the proofs of Theorems 1.1 and 1.2. The proofs are short and simple, but are based on a deep result of Furstenberg and Katznelson. Indeed, the main contribution here is to point out the connection of this theorem to the questions about $\epsilon$-nets. The proof of Theorem 1.3 is described in Sect. 3. It is similar to that of the previous two theorems, but requires an additional ingredient. The final section contains some concluding remarks and open problems.

\section{Strong Nets}

We need a powerful combinatorial result of Furstenberg and Katznelson [16, 17], known as the density Hales-Jewett Theorem. For an integer $k \geq 2$, put $[k]=$ $\{1,2, \ldots, k\}$ and let $[k]^{d}$ denote the set of all vectors of length $d$ with coordinates in $[k]$. A combinatorial line is a subset $L \subset[k]^{d}$ so that there is a set of coordinates $I \subset[d]=\{1,2, \ldots, d\}, I \neq[d]$, and values $k_{i} \in[k]$ for all $i \in I$ for which $L$ is the following set of $k$ members of $[k]^{d}$ :

$$
L=\left\{\ell_{1}, \ell_{2}, \ldots, \ell_{k}\right\}
$$

where

$$
\ell_{j}=\left\{\left(x_{1}, x_{2}, \ldots, x_{d}\right): x_{i}=k_{i} \text { for all } i \in I \text { and } x_{i}=j \text { for all } i \in[d] \backslash I\right\} .
$$

Thus a combinatorial line is a set of $k$ vectors all having some fixed values in the coordinates in $I$, where the $j$ th vector has the value $j$ in all other coordinates. In this notation, the Furstenberg-Katznelson Theorem is the following.

Theorem 2.1 [17] For any fixed integer $k$ and any fixed $\delta>0$, there exists an integer $d_{0}=d_{0}(k, \delta)$ so that for any $d \geq d_{0}$, any set $Y$ of at least $\delta k^{d}$ members of $[k]^{d}$ contains a combinatorial line.

We will also use the following simple lemma.

Lemma 2.2 For every positive integer $d$ there are $d$ vectors $v_{1}, v_{2}, \ldots, v_{d}$ in the plane so that for every two nontrivial sequences of integers $\left(k_{1}, k_{2}, \ldots, k_{d}\right)$ and $\left(k_{1}^{\prime}, k_{2}^{\prime}, \ldots, k_{d}^{\prime}\right)$, with $\left|k_{i}\right|,\left|k_{i}^{\prime}\right|<k$ for all $i$, the two vectors $\sum_{i} k_{i} v_{i}$ and $\sum_{i} k_{i}^{\prime} v_{i}$ have the same direction if and only if $\left(k_{1}, k_{2}, \ldots, k_{d}\right)$ and $\left(k_{1}^{\prime}, k_{2}^{\prime}, \ldots, k_{d}^{\prime}\right)$ have the same direction (that is, one is a multiple of the other). Moreover, there are such vectors $v_{i}$ in which all coordinates are integers of absolute value at most $(2 k-1)^{2 d}$. 
Proof We show that if $v_{i}=\left(x_{i}, y_{i}\right)$ and each of the $2 d$ numbers $x_{i}, y_{i}$ is chosen randomly, uniformly and independently among the set of integers of absolute value at most $(2 k-1)^{2 d}$, then with positive probability the vectors obtained satisfy the desired properties. To prove this is indeed the case, fix two sequences $\left(k_{1}, k_{2}, \ldots, k_{d}\right)$ and $\left(k_{1}^{\prime}, k_{2}^{\prime}, \ldots, k_{d}^{\prime}\right)$, with $\left|k_{i}\right|,\left|k_{i}^{\prime}\right|<k$ and assume they are not proportional. The two vectors $\sum_{i} k_{i}\left(x_{i}, y_{i}\right)$ and $\sum_{i} k_{i}^{\prime}\left(x_{i}, y_{i}\right)$ have the same direction iff

$$
\left(\sum_{i} k_{i} x_{i}\right)\left(\sum_{i} k_{i}^{\prime} y_{i}\right)=\left(\sum_{i} k_{i} y_{i}\right)\left(\sum_{i} k_{i}^{\prime} x_{i}\right),
$$

that is, iff

$$
\sum_{i, j}\left(k_{i} k_{j}^{\prime}-k_{j} k_{i}^{\prime}\right) x_{i} y_{j}=0
$$

As the two vectors $\left(k_{1}, k_{2}, \ldots, k_{d}\right)$ and $\left(k_{1}^{\prime}, k_{2}^{\prime}, \ldots, k_{d}^{\prime}\right)$ are not proportional, the polynomial in the left-hand side of the last equality is nontrivial and has degree 2 . It thus follows, by the Schwartz-Zippel Lemma $[33,36]$ that the probability it vanishes in the random assignment to the variables $x_{i}, y_{i}$ does not exceed $\frac{2}{2 \cdot(2 k-1)^{2 d}+1}<\frac{1}{(2 k-1)^{2 d}}$. Since there are fewer than $(2 k-1)^{2 d}$ choices for the two sequences $\left(k_{1}, k_{2}, \ldots, k_{d}\right)$ and $\left(k_{1}^{\prime}, k_{2}^{\prime}, \ldots, k_{d}^{\prime}\right)$ it follows that with positive probability none of the relevant polynomials vanishes, completing the proof.

Proof of Theorem 1.1 Given a large positive constant $C$, fix an integer $k$ satisfying $k>2 C$, let $d=d_{0}(k, 1 / 2)$ be as in Theorem 2.1 and define $n=k^{d}, \epsilon=\frac{k}{k^{d}}$. Let $v_{1}, v_{2}, \ldots, v_{d}$ be $d$ vectors in $R^{2}$ satisfying the assertion of Lemma 2.2.

Let $X$ be the following set of $k^{d}$ points in the plane.

$$
X=\left\{m_{1} v_{1}+m_{2} v_{2}+\cdots+m_{d} v_{d}: 1 \leq m_{i} \leq k \text { for all } i\right\} .
$$

Note that for every combinatorial line $L$ in $[k]^{d}$, the set of $k$ points

$$
\left\{m_{1} v_{1}+m_{2} v_{2}+\cdots+m_{d} v_{d}:\left(m_{1}, m_{2}, \ldots, m_{d}\right) \in L\right\}
$$

lies on a (geometric) line containing exactly $k$ points of $X$. Indeed, if the combinatorial line is determined by the set of coordinates $I \subset[d]$, then the direction of the corresponding geometric line is $\sum_{i \in[d]-I} v_{i}$, and the generic choice of the vectors $v_{i}$ ensures that this geometric line does not contain any additional points of $X$. Indeed, if $m_{1} v_{1}+m_{2} v_{2}+\cdots+m_{d} v_{d}$ is one of the points of the line, then for any other point $m_{1}^{\prime} v_{1}+m_{2}^{\prime} v_{2}+\cdots+m_{d}^{\prime} v_{d}$ on it, the difference $\left(m_{1}-m_{1}^{\prime}\right) v_{1}+\left(m_{2}-m_{2}^{\prime}\right) v_{2}+$ $\cdots+\left(m_{d}-m_{d}^{\prime}\right) v_{d}$ must have the same direction as $\sum_{i \in[d]-I} v_{i}$. This implies, by the choice of the vectors $v_{i}$, that the vector $\left(m_{1}-m_{1}^{\prime}, m_{2}-m_{2}^{\prime}, \ldots, m_{d}-m_{d}^{\prime}\right)$ is proportional to the characteristic vector of the set $[d]-I$, implying that the vector $\left(m_{1}^{\prime}, m_{2}^{\prime}, \ldots, m_{d}^{\prime}\right)$ also lies in the combinatorial line $L$ and showing that there are exactly $k$ points of $X$ on the corresponding geometric line, as claimed.

It thus follows, by Theorem 2.1 and the choice of $d$, that any set of half the points of $X$ fully contains one of these lines and thus its complement is not an $\epsilon$-net for lines 
for the set $X$, by the definition of $\epsilon$. Therefore, the smallest possible size of such an $\epsilon$-net is bigger than

$$
\frac{1}{2} k^{d}=\frac{k}{2} \frac{1}{\epsilon}>C \cdot \frac{1}{\epsilon} .
$$

This completes the proof of Theorem 1.1.

Proof of Theorem 1.2 The construction is a simple modification of the previous one. Given $C$, pick an integer $k>3 C$. Let $d_{0}=d_{0}(k, 1 / 2)$ be as in Theorem 2.1. For each $d \geq d_{0}$ define $\epsilon=\epsilon(d)=0.9 \frac{k}{k^{d}}$ and let $X$ be a set of $k^{d}$ points in the plane defined as in the previous proof. Thus, any $\epsilon$-net for lines for $X$ contains at least $\frac{1}{2} k^{d}$ points of $X$. For each $n>20 \cdot k^{d}$, let $Y_{n}$ be a set of $n$ points obtained from $X$ by replacing each point $x$ of $X$ by a set $S_{x}$ of either $\lfloor n /|X|\rfloor$ or $\lceil n /|X|\rceil$ points, all very close to $x$. The points in each such set $S_{x}$ are chosen sufficiently close to $x$ to ensure that for every collection of $k$ sets that replace the points corresponding to those of a combinatorial line, there is a fat line containing all the points in these sets, and no other points of $Y_{n}$. Any subset of less than $\frac{1}{2} k^{d}$ of the points in $Y_{n}$ must completely miss at least half of the sets $S_{x}$, and hence, by Theorem 1.1, does not intersect at least one fat line corresponding to a combinatorial line. As each such fat line is of relative size at least

$$
\frac{k\lfloor n /|X|\rfloor}{n}>0.9 \frac{k}{|X|}=\epsilon,
$$

this completes the proof.

\section{Weak Nets}

As mentioned in the introduction, the results in Theorem 1.1 and in Theorem 1.2 can be extended to weak nets as well. The proofs are similar to the case of strong nets but require an additional argument. We proceed with the details.

Proof of Theorem 1.3 Given a large positive $C$, fix an integer $k$ satisfying $k>4 C$, let $d=d_{0}(k, 1 / 2)$ be as in Theorem 2.1, and let $n=k^{d}, \epsilon=\frac{k}{k^{d}}$ be as in the proof of Theorem 1.1. We need the following simple fact.

Claim Consider the set $[k]^{d}$ as a subset of the $d$-dimensional Euclidean space. Call a (geometric) line $L$ in $R^{d}$ special if it contains all $k$ points of a combinatorial line, as defined in Sect. 2. Then the only points of $R^{d}$ that belong to at least two distinct special lines are the members of $[k]^{d}$.

Proof of Claim Let

$$
L_{1}=\left\{\left(x_{1}, x_{2}, \ldots, x_{d}\right)+t \chi_{J_{1}}: t \in(-\infty, \infty)\right\}
$$

and

$$
L_{2}=\left\{\left(y_{1}, y_{2}, \ldots, y_{d}\right)+t \chi_{J_{2}}: t \in(-\infty, \infty)\right\}
$$


be two distinct intersecting special lines, where $\left(x_{1}, x_{2}, \ldots, x_{d}\right),\left(y_{1}, y_{2}, \ldots, y_{d}\right) \in$ $[k]^{d}, J_{1}, J_{2}$ are nonempty subsets of $[d], \chi_{J_{i}}$ is the characteristic vector of $J_{i}, x_{j}=1$ for all $j \in J_{1}$ and $y_{j}=1$ for all $j \in J_{2}$. Note that the definition of a combinatorial line implies that there are such points $\left(x_{1}, x_{2}, \ldots, x_{d}\right)$ and $\left(y_{1}, y_{2}, \ldots, y_{d}\right)$, since any combinatorial line contains a point in $[k]^{d}$ in which all coordinates that are not fixed along the line are equal to $j$, for any desired $j \in[k]$ and in particular for $j=1$.

As the lines are intersecting, there are two reals $t_{1}, t_{2}$ so that

$$
\left(x_{1}, x_{2}, \ldots, x_{d}\right)+t_{1} \chi_{J_{1}}=\left(y_{1}, y_{2}, \ldots, y_{d}\right)+t_{2} \chi_{J_{2}} \text {. }
$$

Since the lines are distinct and intersecting, they are not parallel, and hence the two sets $J_{1}, J_{2}$ determining their directions differ. Without loss of generality, there is an index $j \in J_{1} \backslash J_{2}$. Equating the values in coordinate number $j$ of the two vectors in (1) we conclude that $x_{j}+t_{1}=1+t_{1}=y_{j}$, implying that $1+t_{1} \in[k]$. This implies that each coordinate of the common point $\left(x_{1}, x_{2}, \ldots, x_{d}\right)+t_{1} \chi_{J_{1}}$ of the two lines is in $[k]$, proving the claim.

Returning to the proof of the theorem, let $v_{1}, v_{2}, \ldots, v_{d}$ be random vectors (in the continuous square $[0,1]^{2}$, for example), and define $X$ as in the proof of Theorem 1.1. Call a planar line special if it is the image of a special geometric line in $R^{d}$ under the linear transformation that maps the $i$ th unit vector in $R^{d}$ to $v_{i}$, for all $i$.

It is not difficult to check that the assertion of the last claim and the random choice of the vectors $v_{i}$ imply that with probability 1 every special planar line contains exactly $k$ points of $X$, and the only points in the plane that lie in more than two special planar lines are the points of $X$. This enables one to replace any weak $\epsilon$-net $Y$ that intersects all those special planar lines (ignoring all other lines) by a strong $\epsilon$ net for these lines, of size at most $2|Y|$. Therefore, by the argument in the proof of Theorem 1.1, $2|Y| \geq \frac{1}{2} k^{d}$, implying that $|Y| \geq \frac{1}{4} k^{d}=\frac{k}{4} \frac{1}{\epsilon}>C \cdot \frac{1}{\epsilon}$, as needed. This completes the proof. The derivation of the analog of Theorem 1.2 for weak nets from the result for lines follows by essentially repeating the arguments used in the proof of Theorem 1.2.

\section{Concluding Remarks}

- The proof of [17] applies topological arguments and is not effective, providing no explicit bounds. Subsequent proofs, and in particular the one in [30], do provide some (very weak) estimates, and we can thus write some (extremely slowly), explicit growing function $w$ so that the assertions of Theorems 1.1 and 1.2 hold when $C$ is replaced by $w\left(\left\lceil\frac{1}{\epsilon}\right\rceil\right)$. Indeed, the proof in [30] gives roughly the bound $A_{k}(1 / \delta)$ for the function $d_{0}(k, \delta)$ defined in Theorem 2.1, where $A_{k}$ is the $k$ th function in the Ackermann hierarchy defined recursively as follows: $A_{k}(1)=2$ and $A_{k}(n)=A_{k-1}\left(A_{k}(n-1)\right)$, with $A_{1}(n)=2 n$. Thus, the $k$ th function is obtained by iterating the $(k-1)$ st function, so $A_{2}(n)$ is the exponential function $2^{n}$ and $A_{3}(n)$ is the tower function. Plugging in the proof of Theorems 1.1, 1.2 or 1.3, we conclude that the lower bound they provide is of the form $\Omega\left(\frac{1}{\epsilon} w\left(\left\lceil\frac{1}{\epsilon}\right\rceil\right)\right)$, where $w(s)$ is the minimum number $k$ so that $k^{A_{k}(2)}>s$. 
- The density Hales-Jewett Theorem of Furstenberg and Katznelson is a far reaching strengthening of the Hales-Jewett Theorem [18] that asserts that for any fixed $k$ and $\ell$ there exists a $d_{0}=d_{0}(k, \ell)$ so that for any $d \geq d_{0}$ any coloring of $[k]^{d}$ by $\ell$ colors contains a monochromatic combinatorial line. The Hales-Jewett Theorem is applied in [29], using a similar construction to the one described here, to prove the existence of an infinite collection of lines in the plane so that each point in the plane is covered by at least $k$ lines, and yet in any partition of the lines into two disjoint sets, there is always a point that is not covered by the lines in one of these sets. Note that this only requires the original Hales-Jewett Theorem, and not its stronger density version.

- The example described in Theorem 1.2 clearly implies the same lower bound for several similar range spaces, like the one in which the objects are points in the plane and the ranges are planar rectangles or triangles.

- An equivalent concise way of describing the proof of Theorem 1.1 is the following. Consider the set $Z=[k]^{d}$ as a subset of the Euclidean space $R^{d}$. As in the proof of Theorem 1.3 call a (geometric) line in this space special if it contains all $k$ points of one of the combinatorial lines defined in Sect. 2. Put $\epsilon=\frac{k}{k^{d}}$. The FurstenbergKatznelson result (Theorem 2.1) implies that if $d$ is sufficiently large, then the minimum possible size of an $\epsilon$-net for $Z$ with respect to the range space consisting of all special lines is at least $\frac{1}{2} k^{d}$. Now map $Z$ by a random linear transformation to the plane $R^{2}$, and note that with probability 1 no two points of $Z$ are mapped to the same point, and every special line is mapped to a line containing exactly $k$ points of the image of $Z$. (This is proved algebraically in Lemma 2.2.) Let $X$ be the image of $Z$. The smallest size of an $\epsilon$-net for $X$ is at least $\frac{1}{2} k^{d}$, as the set of points of $Z$ mapped to any such net intersects all special lines.

The result for weak nets (Theorem 1.3) follows in a similar way. As shown in the Claim appearing in the proof of this theorem, the only points in $R^{d}$ that are common to at least two special lines are the points of $Z$, and thus any weak net for these lines in $R^{d}$ can be converted into a strong net of the same cardinality, which has to be large, by Theorem 2.1. A random projection to $R^{2}$ maps $Z$ to a set of points $X$ and maps each special line to a line we call a special planar line. Moreover, with probability 1 the only points of the plane that belong to more than 2 of the special planar lines are the points of $X$. Thus for any weak $\epsilon$-net $Y$ for $X$ in the plane with respect to the special planar lines, its inverse image in the set of all points belonging to at least one special line in $R^{d}$ is of size at most $2|Y|$ and forms a weak $\epsilon$-net for $Z$ with respect to the set of all special lines. This shows that if $d$ is sufficiently large, then $2|Y| \geq \frac{1}{2} k^{d}$, providing the required estimate.

- It may be a bit better in terms of the estimates obtained to consider Moser numbers rather than Hales-Jewett numbers (cf. [31] for the definition of Moser numbers). This will still provide very poor (and yet non-linear) lower bounds.

- The problem of deciding whether or not there are natural geometric range spaces of VC-dimension $d$ in which the minimum possible size of an $\epsilon$-net is $\Omega\left(\frac{d}{\epsilon} \log \left(\frac{1}{\epsilon}\right)\right)$ remains open. It seems plausible to conjecture that there are such examples, and even to speculate that this is the case for the range space of lines in the plane, for appropriately defined planar sets of points. 
Acknowledgement I would like to thank Esther Ezra and Shakhar Smorodinsky for helpful discussions, and János Pach for telling me about [29].

Note Added in Proof Very recently, Pach and Tardos proved that there are geometric range spaces of VC-dimension 2 in which the minimum possible size of an $\epsilon$-net is $\Omega\left(\frac{1}{\epsilon} \log \left(\frac{1}{\epsilon}\right)\right)$. Their method does not seem to provide any non-linear bounds for weak $\epsilon$-nets.

\section{References}

1. Agarwal, P.K.: Partitioning arrangements of lines I. Discrete Comput. Geom. 5, 449-483 (1990)

2. Agarwal, P.K.: Partitioning arrangements of lines II. Discrete Comput. Geom. 5, 553-573 (1990)

3. Alon, N., Spencer, J.H.: The Probabilistic Method, 3rd edn. Wiley, New York (2008). xv+352 pp.

4. Alon, N., Haussler, D., Welzl, E.: Partitioning and geometric embedding of range spaces of finite Vapnik-Chervonenkis dimension. In: Proc. of the Third Annual Symposium on Computational Geometry, Waterloo, Canada, pp. 331-340. ACM, New York (1987)

5. Alon, N., Bárány, I., Füredi, Z., Kleitman, D.J.: Point selections and weak $\epsilon$-nets for convex hulls. Comb. Probab. Comput. 1, 189-200 (1992)

6. Alon, N., Kalai, G., Matoušek, J., Meshulam, R.: Transversal numbers for hypergraphs arising in geometry. Adv. Appl. Math. 29, 79-101 (2002)

7. Aronov, B., Ezra, E., Sharir, M.: Small-size epsilon-nets for axis-parallel rectangles and boxes. In: Proc. STOC 09, pp. 639-648

8. Brönnimann, H., Goodrich, M.T.: Almost optimal set covers in finite VC dimensions. Discrete Comput. Geom. 14, 463-479 (1995)

9. Bukh, B., Matoušek, J., Nivasch, G.: Lower bounds for weak epsilon-nets and stair-convexity. In: Proc. 25th ACM Symp. on Computational Geometry (SoCG 2009), pp. 1-10 (2009)

10. Chazelle, B., Friedman, J.: A deterministic view of random sampling and its use in geometry. Combinatorica 10, 229-249 (1990)

11. Chazelle, B., Edelsbrunner, H., Grigni, M., Guibas, L.J., Sharir, M., Welzl, E.: Improved bounds on weak $\epsilon$-nets for convex sets. Discrete Comput. Geom. 13, 1-15 (1995)

12. Clarkson, K.: New applications of random sampling in computational geometry. Discrete Comput. Geom. 2, 195-222 (1987)

13. Clarkson, K.L., Varadarajan, K.: Improved approximation algorithms for geometric set cover. Discrete Comput. Geom. 37, 43-58 (2007)

14. Even, G., Rawitz, D., Shahar, S.: Hitting sets when the VC-dimension is small. Inf. Process. Lett. 95, 358-362 (2005)

15. Füredi, Z., Pach, J.: Traces of finite sets: extremal problems and geometric applications. In: Extremal problems for finite sets, Visegrád, 1991. Bolyai Soc. Math. Stud., vol. 3, pp. 251-282. János Bolyai Math. Soc., Budapest (1994)

16. Furstenberg, H., Katznelson, Y.: A density version of the Hales-Jewett theorem for $k=3$. In: Graph Theory and Combinatorics, Cambridge, 1988. Discrete Math., vol. 75, pp. 227-241 (1989)

17. Furstenberg, H., Katznelson, Y.: A density version of the Hales-Jewett theorem. J. Anal. Math. 57, 64-119 (1991)

18. Hales, A.W., Jewett, R.I.: Regularity and positional games. Trans. Am. Math. Soc. 106, 222-229 (1963)

19. Har-Peled, S., Kaplan, H., Sharir, M., Smorodinsky, S.: $\epsilon$-nets for half-spaces revisited. Manuscript (2008)

20. Haussler, D., Welzl, E.: $\epsilon$-nets and simplex range queries. Discrete Comput. Geom. 2, 127-151 (1987)

21. King, J., Kirkpatrick, D.G.: Improved approximation for guarding simple galleries from the perimeter. arXiv:1001.4231 (2010)

22. Komlós, J., Pach, J., Woeginger, G.: Almost tight bounds for epsilon nets. Discrete Comput. Geom. 7, 163-173 (1992)

23. Matoušek, J.: Reporting points in half-spaces. Comput. Geom. 2, 169-186 (1992)

24. Matoušek, J.: Lectures on Discrete Geometry. Graduate Texts in Mathematics, vol. 212. Springer, New York (2002). xvi+481 pp.

25. Matoušek, J., Wagner, U.: New constructions of weak $\epsilon$-nets. Discrete Comput. Geom. 32, 195-206 (2004) 
26. Matoušek, J., Seidel, R., Welzl, E.: How to net a lot with little: small $\epsilon$-nets for disks and halfspaces. In: Proc. 6th Annu. ACM Sympos. Comput. Geom., pp. 16-22 (1990). Revised version at http://kam.mff.cuni.cz/matousek/enets3.ps.gz

27. Pach, J., Agarwal, P.K.: Combinatorial Geometry. Wiley-Interscience Series in Discrete Mathematics and Optimization, A Wiley-Interscience Publication. Wiley, New York (1995). xiv+354 pp.

28. Pach, J., Woeginger, G.: Some new bounds for $\epsilon$-nets. In: Proc. 6th Annual Symposium on Computational Geometry, pp. 10-15. ACM, New York (1990)

29. Pach, J., Tardos, G., Tóth, G.: Indecomposable coverings. Can. Math. Bull. 52(3), 451-463 (2009)

30. Polymath, D.H.J.: A new proof of the density Hales-Jewett theorem. arXiv:0910.3926

31. Polymath, D.H.J.: Density Hales-Jewett and Moser numbers. arXiv:1002.0374

32. Pyrga, E., Ray, S.: New existence proofs for $\epsilon$-nets. In: Proc. 24th Annu. ACM Sympos. Comput. Geom., pp. 199-207 (2008)

33. Schwartz, J.: Fast probabilistic algorithms for verification of polynomial identities. Journal of the ACM, 701-717 (1980)

34. Vapnik, V.N., Chervonenkis, A.Yu.: On the uniform convergence of relative frequencies of events to their probabilities. Theory Probab. Appl. 16, 264-280 (1971)

35. Varadarajan, K.R.: Epsilon nets and union complexity. In: Symposium on Computational Geometry, pp. 11-16 (2009)

36. Zippel, R.: Probabilistic algorithms for sparse polynomials. In: Symbolic and Algebraic Computation (EUROSAM'79, Internat. Sympos.), Marseille, 1979. Lecture Notes in Comput. Sci., vol. 72, pp. 216226. Springer, Berlin (1979) 